\title{
Monte Carlo Study of 3D Stray Radiation during Interventional Procedures
}

\author{
Khalid S. Alzimami \\ Department of Radiological Sciences, College of Applied Medical Sciences, King Saud University, Riyadh, KSA \\ Email: kalzimami@ksu.edu.sa
}

How to cite this paper: Alzimami, K.S (2018) Monte Carlo Study of 3D Stray Radiation during Interventional Procedures. International Journal of Medical Physics, Clinical Engineering and Radiation Oncology, 7, 453-463.

https://doi.org/10.4236/ijmpcero.2018.74038

Received: August 28, 2018

Accepted: October 5, 2018

Published: October 8, 2018

Copyright (c) 2018 by author and Scientific Research Publishing Inc. This work is licensed under the Creative Commons Attribution International License (CC BY 4.0).

http://creativecommons.org/licenses/by/4.0/

\begin{abstract}
In interventional medical procedures, other than the highly important issue of optimizing image quality and patient exposure using the primary beam, there remains a continuing need for the study of staff exposure from the scattered radiation. Herein, investigation is made of the 3D stray-radiation distribution, the simulation being made of a realistic interventional scenario through use of the Monte Carlo code Geant4 (version 10.3). The simulation is conducted based on the high definition reference Korean-man (HDRK-man) computational phantom and a GE Infinia 3/8" C-arm machine, focusing on the effect of variation of $\mathrm{kVp}$ and field of view (FoV) on the scattered particles' spatial distribution. With direct measurement of the absorbed dose remaining challenging, not least in respect of the organs at risk, we computed the scatter fractions, defined as the ratio of the air kerma free-in-air to the entrance surface air kerma (ESAK), which are both easily quantifiable. Scatter fraction distributions were simulated for X-ray tube outputs (and half-value layers, HVL) of $60 \mathrm{kVp}(2.3 \mathrm{~mm} \mathrm{Al}), 80 \mathrm{kVp}(3.2 \mathrm{~mm} \mathrm{Al})$ and $120 \mathrm{kVp}(4.3$ $\mathrm{mm} \mathrm{Al}$ ) and $\mathrm{FoV}$ of 15, 20, 25 and $30 \mathrm{~cm}$. The distributions are obtained for different height levels, corresponding to the lens of the eye, and the lung and prostate, all radiosensitive organs. Investigations are made for eight likely locations around the patient. At fixed FoV results reveal an inverse relationship between ESAK and $\mathrm{kVp}$, also that change in $\mathrm{kVp}$ from 60 to 80 has a greater effect than from 80 to 120 . For change in FoV at fixed $k V p$, the scatter fraction remains constant. The particular staff locations are found to be optimal in seeking mitigation of dose. Moreover, the combined usage of numerical human model and Monte Carlo simulation can be considered as an added value to the radiation safety research field, especially to the interventional radiology staff and to the patient.
\end{abstract}

\section{Keywords}

Stray Radiation, Interventional Procedures, Operator Exposure, Geant4 


\section{Introduction}

Interventional radiology (IR) and cardiology (IC) are of undoubted value in the diagnosis and treatment of diseases, their efficacy being reflected in the growing availability of these procedures within many larger medical centers throughout the world. The procedures are conducted by a team of medical staff who during the intervention all remain within the specialized theatre, typically being in close proximity to the patient. Given that such live-time imaging procedures are typically of protracted duration, it is of no surprise that when compared to other frequent radiological methods, IR and IC represent two of the most significant in terms of radiation exposure to medical staff. Although perhaps obvious, it is nevertheless worth noting that with the ever-increasing demand for the conduct of such procedures there comes an associated increase in radiation exposure to staff. A priori knowledge of the occupational absorbed dose to such staff can assist in keeping exposure levels as low as reasonably achievable [1]. Of interest is that previous success in maintaining levels below the dose limit has not always prevented cataract formation, with incidences of brain cancer among radiologists also being suggested as being implicated [2] [3] [4] [5] [6]. As such, computational-exposure scenarios mimicking realistic interventional procedures are important in predicting the 3D scatter (stray) radiation arising from the patient and apparatus. Over the last decade, investigations of patient and staff exposure during IR and IC have been performed via measurement [7] [8] [9] [10] [11]. Study of interventional radiology occupational exposures has also been made using the Monte Carlo (MC) technique [12] [13] [14]. This said, the stray radiation 3D distribution has not been previously obtained via $\mathrm{MC}$ simulation using the high definition reference Korean-man (HDRK-man) virtual anthropomorphic phantom [15]. Herein modeling has been made of staff exposure, the patient being imaged with a GE Infinia 3/8" machine, utilizing the versatile Geant4 toolkit [16]. The aim has been to study the effect of key parameters on occupational exposure distribution within the theatre. Specifically, the influence of $\mathrm{kVp}$, field of view ( FoV) and staff position has been investigated, focusing on three radiosensitive organs (lens of the eye, and lung and prostate).

\section{Materials and Methods}

Description is provided herein of the adopted methodology, presenting the voxelized phantom corresponding to the patient and staff, the X-ray imaging machine and X-ray tube output. Different proposed interventional imaging procedure scenarios are also outlined. We note that there are three phantom format types (stylized, voxel and hybrid) and four morphometric categories (reference, patient-dependent, patient-sculpted and patient-specific). Here we are limited to voxel type and reference category (patient matching by age only) to model phantoms.

\subsection{Patient and Operator Modeling}

In modeling patient and staff, close similarity has been noted between the ICRP 
reference man and the voxelized phantom HDRK-man, including height and weight, a matter encouraging the use of the latter. Constructed from high-resolution photographic anatomical images, HDRK-man has a height of $171 \mathrm{~cm}$ and weight of $68 \mathrm{~kg}$. Whereas, the ICRP models human having a height of $176 \mathrm{~cm}$ and weight of $73 \mathrm{~kg}$. The considered phantom consists of some 30 million voxels, resolution $1.981 \times 1.981 \times 2.0854 \mathrm{~mm}^{3}$, distributed along a 3D array of dimension $247 \times 141 \times 850$, along the $\mathrm{x}, \mathrm{y}$ and $\mathrm{z}$ coordinate axes, respectively. The elemental composition and density of each voxel were obtained from ICRU 46 [17], defining some 40 tissues/organs.

\subsection{Gamma Camera and X-Ray Spectra}

The GE Infinia II 3/8" C-arm machine modeled in this study has an inner radius of $1 \mathrm{~m}$ and is made from a sturdy, rigid foam material (density $0.7 \mathrm{~g} / \mathrm{cm}^{3}$ and elemental composition of $50 \% \mathrm{H}, 30 \% \mathrm{C}, 10 \% \mathrm{~N}$ and $10 \% \mathrm{O}$ ). As shown in Figure 1, the gantry supports a CsI flat panel imager $\left(48 \times 48 \times 10 \mathrm{~cm}^{3}\right)$ positioned close to the upper surface of the patient, while the under-couch X-ray tube is contained in an iron shielding surround. The couch is a $45 \times 190 \times 1.5 \mathrm{~cm}^{3}$ table made of PMMA material. A typical beam-hardening $\mathrm{Pb}$ sheet of $0.5 \mathrm{~mm}$ thickness has been added to the patient table in front of the primary X-ray beam. In generating the X-ray tube outputs, as modified by the particular choice of total filtration and parameter selection $(\mathrm{kVp}$ and $\mathrm{mAs})$, use was made of the software known as spektr [18].

\subsection{Irradiation Scenarios}

The parameters studied during this work were as follows:

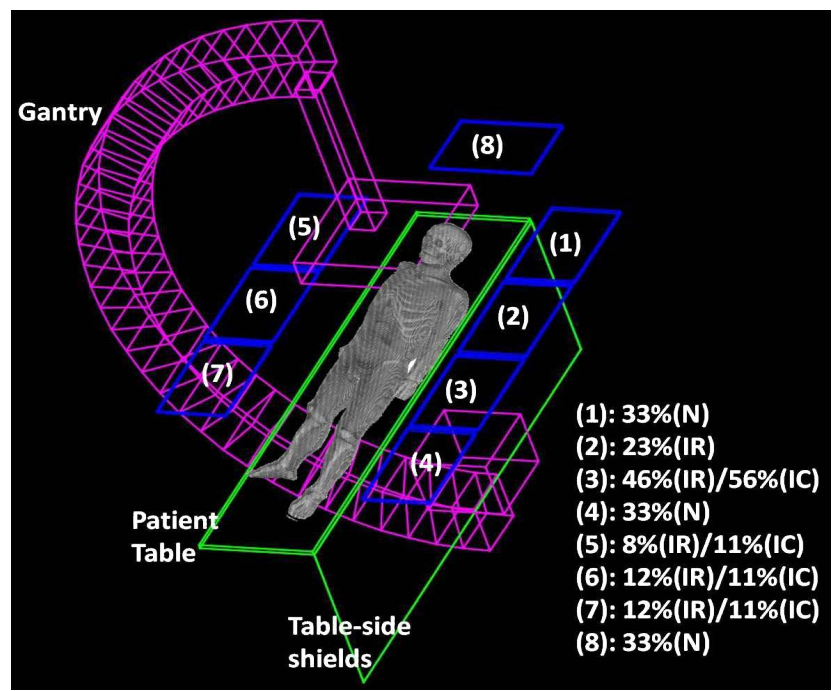

Figure 1. Simulated experimental set-up, including the C-arm machine (Gantry, X-ray tube holder, CsI flat panel) and the HDRK-Man phantom lying on a table shielded with $0.5 \mathrm{~mm} \mathrm{~Pb}$ on its left side. Also shown: the eight most likely occupied locations by staff (Interventional Radiologist (IR), Interventional Cardiologist (IC) and Nurse (N)) with their occurrence (in \%) during an interventional procedure. 
- X-ray photon spectra: $60 \mathrm{kVp}$ (Half Value Layer $(\mathrm{HVL})=2.3 \mathrm{~mm} \mathrm{Al}$ ), 80 $\mathrm{kVp}(\mathrm{HVL}=3.2 \mathrm{~mm} \mathrm{Al})$ and $120 \mathrm{kVp}(\mathrm{HVL}=4.3 \mathrm{~mm} \mathrm{Al})$

- C-arm orientation (fixed): vertical

- Exposure and patient orientation: postero-anterior (PA), lying on the bed

- Irradiated body area (denoted as FoV) (four values): $15 \times 15,20 \times 20,25 \times$ 25 , and $30 \times 30 \mathrm{~cm}^{2}$, centered at chest

- Staff locations (eight values): about $40 \mathrm{~cm}$ around the patient table, as proposed in literature [19] and shown in Figure 1

- Table height (fixed): $75 \mathrm{~cm}$

\subsection{Simulation and Data Collection}

For MC simulation, use has been made of the Geant 4 version 10.3 multithreaded mode [16] [20]. In respect of the large number of voxels representing the patient, utilization has been made of the nested parameterization provided for by the G4VNested Parameterization class. For the simulated X-ray room, a particular typical example of dimension $4.8 \times 6.4 \times 2.89 \mathrm{~m}^{3}$ was chosen. Spectra generated through use of the spektr software have been included in the Geant4-based program, the inverse transform method being used to randomly generate the corresponding energies. Such method consisting of the sampling of random numbers from a given probability distribution function, uses the inverse cumulative distribution defined by the calculation of the cumulative integration for a given set of samples. For all cases, a pyramidal-shaped primary beam has been assumed, emanating from a point-like X-ray source towards the chest of the patient. In allowing simulation of the spatial distribution of the stray radiation, the room space was subdivided into $80 \times 80 \times 34$ air-filled boxes, each of the dimension $5 \times 5 \times 5 \mathrm{~cm}^{3}$. The scenario allows for scattered radiation evaluation up to 2 $\mathrm{m}$ away from the centre of the irradiation field. For a given choice of set-up, simulation has been made to obtain the air kerma free-in-air within air-filled boxes of the dimensions $10 \times 10 \times 10 \mathrm{~cm}^{3}$, evaluated at the level of the lens of the eye, lung and prostate, at 165,150 and $84 \mathrm{~cm}$ directly above the floor respectively. In order to obtain the parameter ESAK, simulation was made of the absorbed dose evaluated within $10 \times 10 \times 1 \mathrm{~cm}^{3}$ air-filled boxes inserted directly under the patient table; this allows X-ray backscattering to be taken into account. All simulations were carried out on a Dell Precision T7610 workstation with 40 cores, equipped with an Intel Xeon E5-2680v2 CPU at $2.80 \mathrm{GHz}$ and $256 \mathrm{~GB}$ RAM, working on the Linux operating system (Ubuntu 14.04). The number of generated primary particles was $5 \times 10^{8}$ (allowing statistical uncertainty of $<3 \%$ ) for all cases, with run times ranging from $8500 \mathrm{~s}$ to $17560 \mathrm{~s}$ (i.e. from 2.4 hours up to just below 5 hours).

\section{Results and Discussion}

Results and analysis are provided herein, concerning the three dominating parameters modifying the $3 \mathrm{D}$ stray radiation dose distribution, $\mathrm{kVp}$, FoV and staff 
location, evaluated at the level of the eyes, lung and prostate.

\subsection{Effect of $\mathrm{kVp}$}

To assess the influence of $\mathrm{kVp}$ on the simulated scattered radiation distributions, comparison has been made of three cases: $120 \mathrm{kVp}(\mathrm{HVL}=4.3 \mathrm{~mm} \mathrm{Al}), 80 \mathrm{kVp}$ $(\mathrm{HVL}=3.2 \mathrm{~mm} \mathrm{Al})$ and $60 \mathrm{kVp}(\mathrm{HVL}=2.3 \mathrm{~mm} \mathrm{Al})$ for a fixed value of $\mathrm{FoV}$. Figures 2-4 provide dose maps at the particular organ levels. In changing the $\mathrm{kVp}$ from 60 to 80 to 120 , the distribution of stray radiation is seen to progressively concentrate around the irradiated area of the patient.

Such results are in accord with literature [10] and theory, the latter depicted in Figure 5 showing polar plots of the Compton scattering cross-section as a function of energy [21], the forward direction becoming increasingly favored. Table 1 illustrates the ratios of air kerma free-in-air to entrance surface air kerma (ESAK), namely the scatter fraction, quoted in $\mu \mathrm{Gy} / \mathrm{Gy} \cdot \mathrm{cm}^{2}$ [22], for all combinations of the eight staff locations, organ levels, FoV and spectral influence of $\mathrm{kVp}$. As previously mentioned, observation is made of an inverse proportionality between ESAK and $\mathrm{kVp}$. Moreover, in changing the $\mathrm{kVp}$ from 60 to 80 and from 80 to 120, keeping the FoV fixed, the scatter fraction is observed to approximately double, remaining almost constant for the three organ levels and the eight staff locations.

This can be rationalized to be due to the penetration and scattering abilities characterizing the spectral X-ray beam. Given the limited number of interventional scenarios studied, including the overall shape of the phantom, the choice
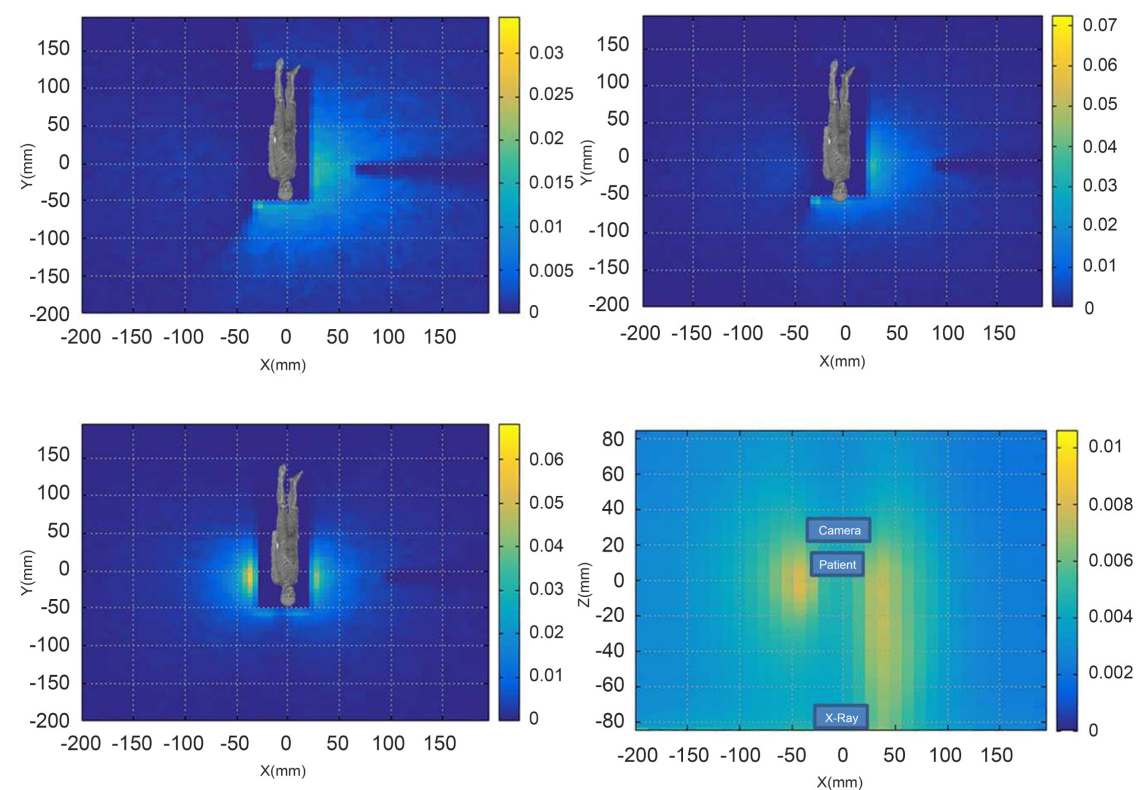

Figure 2. Simulated stray radiation obtained at $120 \mathrm{kVp}(4.3 \mathrm{~mm} \mathrm{Al})$ and $30 \mathrm{~cm} \mathrm{FoV}$. Shown are dose maps for three horizontal plans, corresponding to eye lens (top left), lung (top right) and prostate (bottom left) levels; a vertical plan perpendicular to the C-arm gantry is shown at the bottom right. The colour bar indicates the relative strength of scattered radiation, from lowest values at the blue end to yellow at the upper end of the scale. 

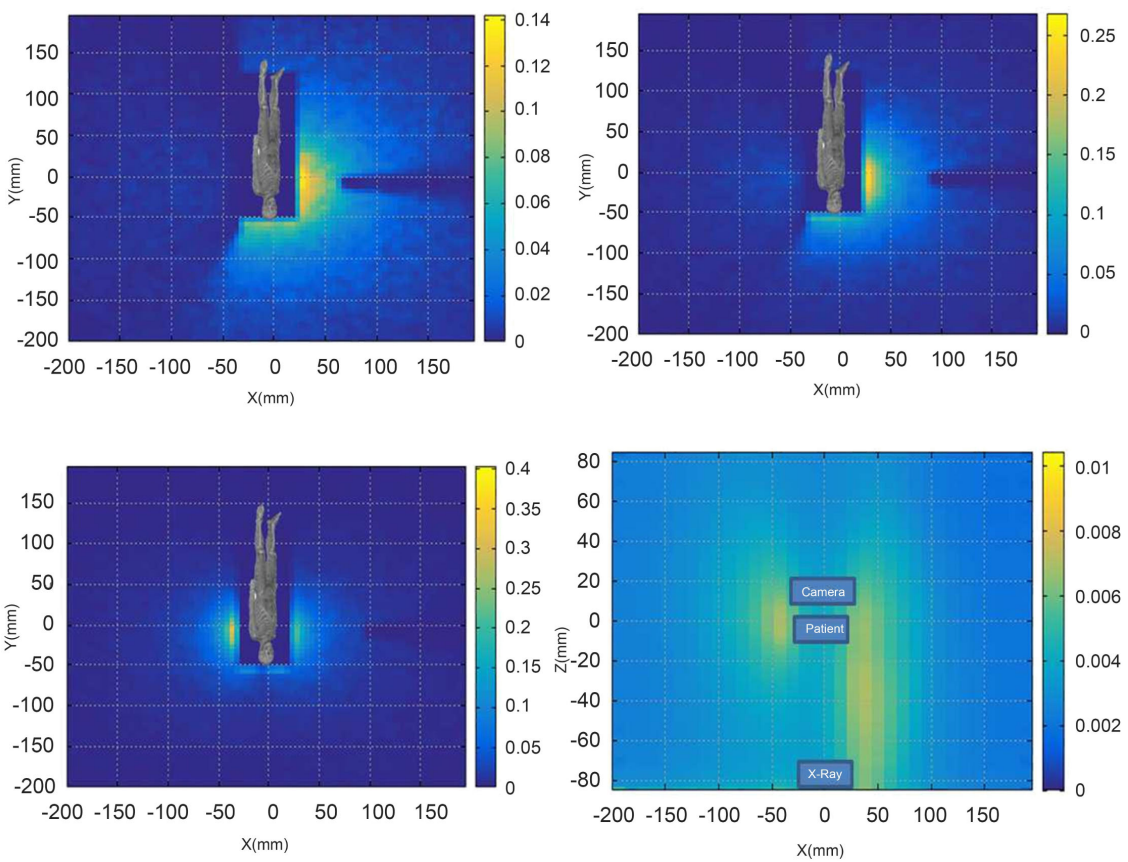

Figure 3. Simulated stray radiation obtained at $60 \mathrm{kVp}(2.3 \mathrm{~mm} \mathrm{Al})$ and $30 \mathrm{~cm} \mathrm{FoV}$. Dose maps are shown for three horizontal plans at the level of the lens of eye (top left), lung (top right) and prostate (bottom left); a vertical plan normal to the C-arm gantry is shown at the bottom right.
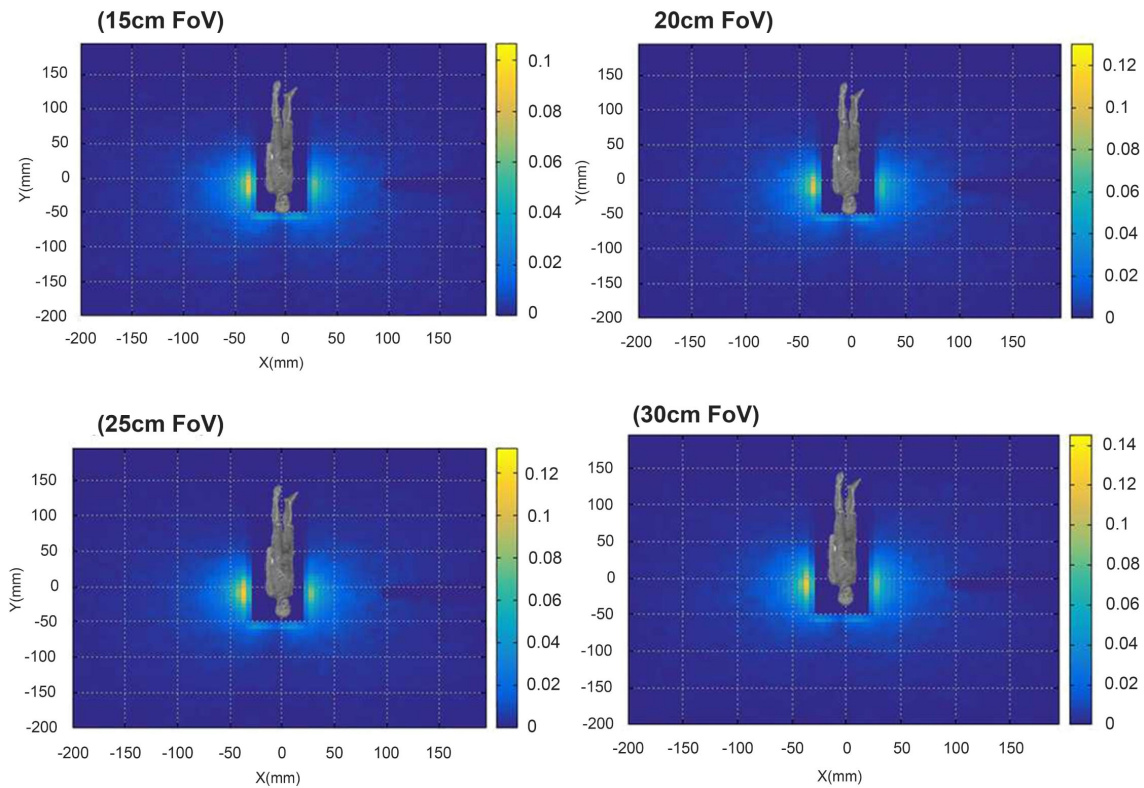

Figure 4. Simulated stray radiation obtained at $80 \mathrm{kVp}(3.2 \mathrm{~mm} \mathrm{Al})$ corresponding to the horizontal patient level when varying the FoV from $15 \mathrm{~cm}$ (top left) to $30 \mathrm{~cm}$ (bottom right).

of $\mathrm{C}$-arm machine and buildup specifications, the findings are not to be generalized. Nevertheless, while each interventional procedure will undoubtedly produce its own scattered radiation distribution, the current study can be considered to provide a good estimation of the overall situation. 


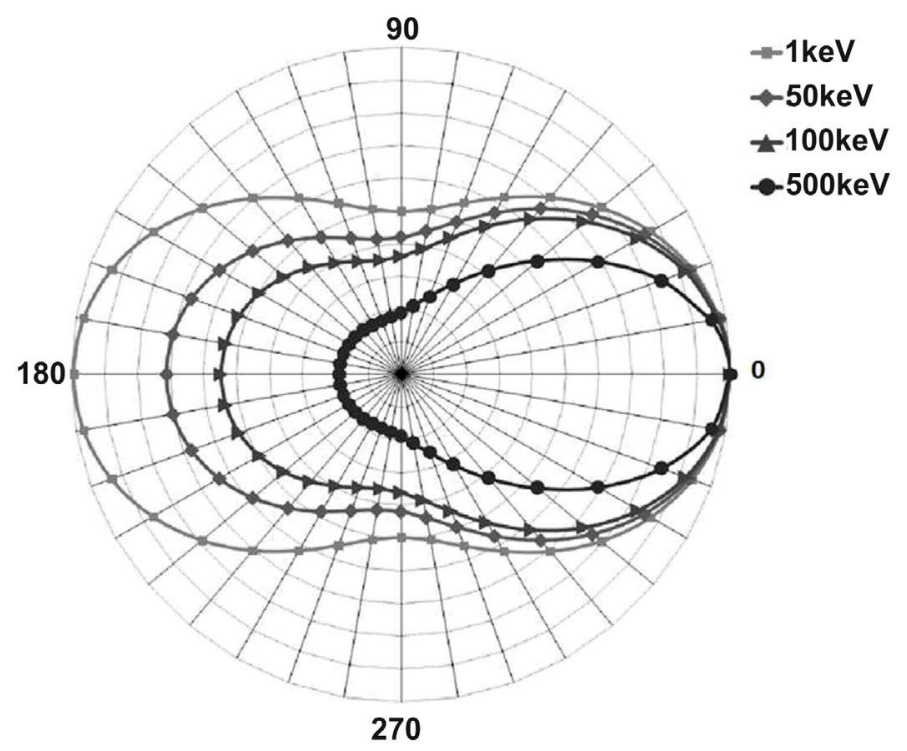

Figure 5. Klein-Nishina differential cross-sections as a function of the scattering angle and incoming photon energies.

Table 1. Simulated scatter fractions (in $\mu \mathrm{Gy} / \mathrm{Gy} \cdot \mathrm{cm}^{2}$ ) at the level of the lens of the eye, and lung and prostate, also the ESAK values. Data are given for all possible combinations of $\mathrm{kVp}(60,80$ and 120) and FoV $(15,20,25$ and $30 \mathrm{~cm})$. The eight most likely occupied locations (Loc.) were emphasized. Cells highlighted in gray correspond to the radiological safe side.

\begin{tabular}{|c|c|c|c|c|c|c|c|c|c|c|c|c|c|}
\hline \multirow[b]{2}{*}{ Loc. } & \multirow[b]{2}{*}{ FoV/Z (cm) } & \multicolumn{4}{|c|}{$60 \mathrm{kVp}$} & \multicolumn{4}{|c|}{$80 \mathrm{kVp}$} & \multicolumn{4}{|c|}{$120 \mathrm{kVp}$} \\
\hline & & 15 & 20 & 25 & 30 & 15 & 20 & 25 & 30 & 15 & 20 & 25 & 30 \\
\hline \multirow{4}{*}{1} & 165 & 1.1 & 0.8 & 3.2 & 7.1 & 2.3 & 2.3 & 4.3 & 5.2 & 1.6 & 2.7 & 2.6 & 3 \\
\hline & 150 & 28.1 & 29.1 & 29 & 30.9 & 40.5 & 36.7 & 37.8 & 27.4 & 42.2 & 37.7 & 34.5 & 37.7 \\
\hline & 84 & 26.1 & 25.6 & 23.6 & 29.8 & 55.8 & 49.4 & 58.9 & 52.5 & 56.9 & 60.2 & 63.3 & 55.9 \\
\hline & Sum & 55.2 & 55.4 & 55.8 & 67.7 & 98.6 & 88.4 & 101 & 85.1 & 100.7 & 100.7 & 100 & 96.6 \\
\hline \multirow{4}{*}{2} & 165 & 1.5 & 2.2 & 4.5 & 5.8 & 2.9 & 2 & 4.7 & 6.3 & 1.8 & 0.1 & 3.8 & 10.4 \\
\hline & 150 & 31.4 & 29 & 30 & 31.4 & 49 & 38.8 & 40.1 & 36.3 & 38.9 & 41.4 & 42.8 & 43.2 \\
\hline & 84 & 34.2 & 33.3 & 33.4 & 36.3 & 59.7 & 68.1 & 69.6 & 65.8 & 77.2 & 73.9 & 69.8 & 82.8 \\
\hline & Sum & 67 & 64.5 & 67.8 & 73.5 & 111.7 & 108.9 & 114.5 & 108 & 117.9 & 115.4 & 116 & 136.4 \\
\hline \multirow{4}{*}{3} & 165 & 2.3 & 4.1 & 8.8 & 8 & 4.8 & 6.2 & 9.4 & 12.3 & 2.9 & 7.1 & 7.9 & 8.1 \\
\hline & 150 & 18.2 & 16.9 & 15.2 & 14.9 & 23.9 & 21.8 & 18.9 & 20.3 & 25.1 & 22 & 21.5 & 22.6 \\
\hline & 84 & 15 & 11.5 & 10.1 & 15 & 33.3 & 32 & 23.8 & 23 & 22.2 & 23.3 & 29.9 & 33.4 \\
\hline & Sum & 35.5 & 32.5 & 34.1 & 37.9 & 62 & 60 & 52.1 & 55.6 & 50.2 & 52.4 & 59.3 & 64 \\
\hline & 165 & 6.2 & 6 & 8.9 & 7.6 & 11.9 & 11.1 & 6.4 & 7.7 & 10 & 12.9 & 11.2 & 12.9 \\
\hline & 150 & 4.7 & 5.3 & 6.9 & 9.5 & 12.9 & 7.6 & 11.4 & 10.2 & 10.1 & 6.3 & 13 & 9.1 \\
\hline & 84 & 6.8 & 4.8 & 3.7 & 4.1 & 13 & 7.5 & 7.7 & 6.6 & 15.9 & 6.3 & 12.2 & 11.7 \\
\hline & Sum & 17.7 & 16.2 & 19.6 & 21.2 & 37.8 & 26.2 & 25.5 & 24.5 & 36.1 & 25.4 & 36.3 & 33.7 \\
\hline 5 & 165 & 0.9 & 2.7 & 2.7 & 6.7 & 2.9 & 4.1 & 5.4 & 7 & 2.9 & 5.6 & 8 & 8.2 \\
\hline
\end{tabular}


Continued

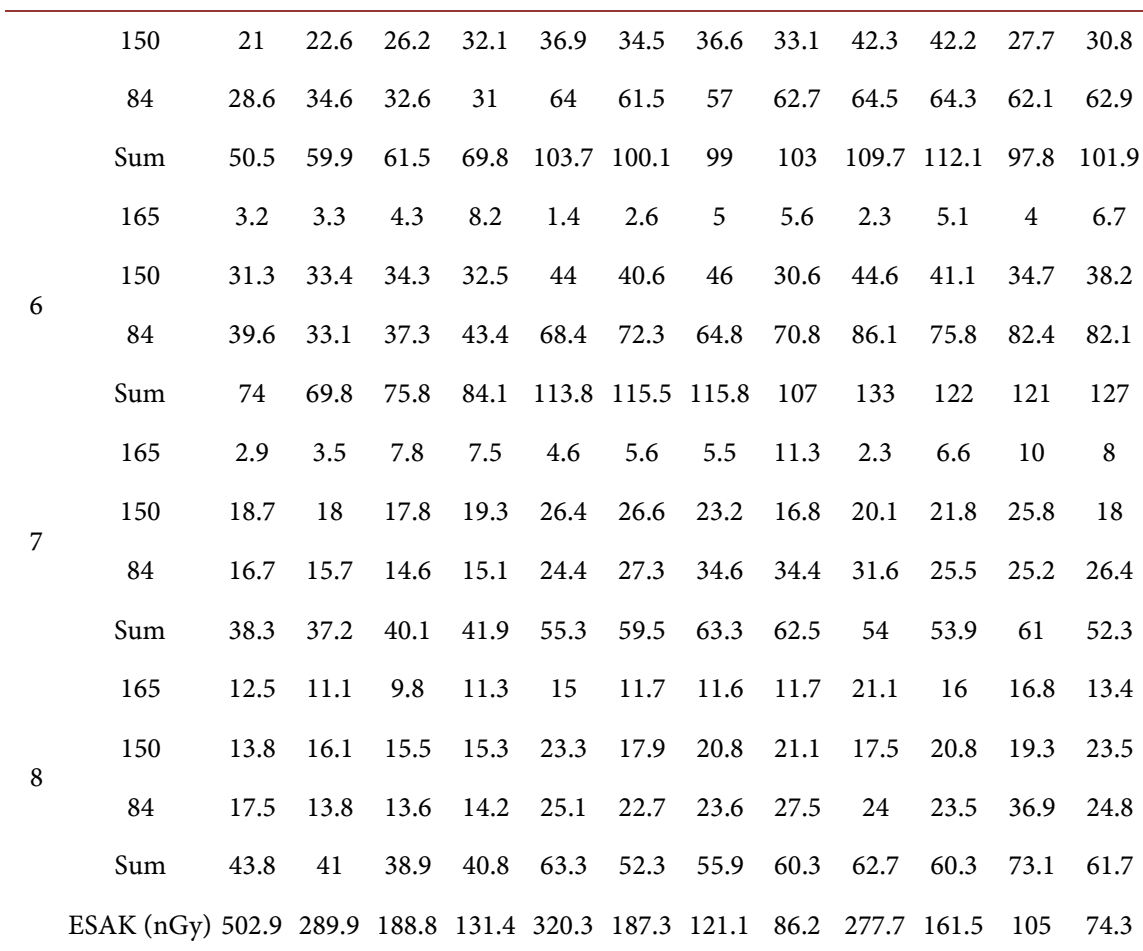

\subsection{Effect of the FoV}

In the example case of $80 \mathrm{kVp}$, stray radiation maps have been provided for particular likely FoV values of 15, 20, 25 and $30 \mathrm{~cm}$. Horizontal plans corresponding to the patient table level are shown in Figure 4. No significant trend is discernible at this level, contrary to the ESAK and the scatter fraction distributions. In changing from a FoV of 15 to $30 \mathrm{~cm}$, ESAK decreases continuously reaching values of a factor of three or better. Although limited to $80 \mathrm{kVp}$ and to 14,20 and $28 \mathrm{~cm}$ circular shaped FoVs, Bethe et al. [23] found this to be an important effect on the stray radiation when changing the FoV parameter; the present work confirms their findings. Also supported is the trend towards near constancy of scatter fractions for a given value of $\mathrm{kVp}$. Thus, we can generalize that the scatter fraction remains an almost constant function of the FoV for a fixed $\mathrm{kVp}$ parameter. This situation should be explored by extending the present study to take into account the effect of focal-to-skin and focal-to-detector distances.

\subsection{Effect of Operator Location}

The effect of changing staff location (as shown in Figure 1) on the scatter fraction at the level of the lens of the eye, lung and prostate is illustrated in Table 1. Despite buildup effects as observed in the measurements, applicability of the inverse square law has been confirmed, in terms of scattered fraction values, for the coupled staff locations of ( 1 and 5), (2 and 6) and ( 3 and 7). Moreover, we observe the typical times spent by the different members of staff (IR, IC and N) during an interventional procedure [19] to be optimal. This said, the present 
study has been limited to the PA projection; further simulations would help, extending findings to other irradiation conditions of AP, LAO, RAO and such like.

\section{Conclusion}

There is an increasingly pressing need to quantify medical staff during interventional procedures. The present study, concerning scattered radiation from the patient and C-arm, has examined the influence of $\mathrm{kVp}$, FoV and staff location on the 3D distribution and dose. Using an anthropomorphic voxelized phantom incorporated into a Geant4-based program, evaluation has been made of ESAK and scatter fraction at three organ levels of members of staff (eye lens, lung and prostate). For a given value of the FoV, ESAK is seen to increase with a decrease in $\mathrm{kVp}$, supporting existing literature. Secondly, modification of $\mathrm{kVp}$ from 120 to 80 has a negligible effect (average deviation of $13.4 \%$ ); conversely, in changing from 80 to $60 \mathrm{kVp}$ an average decrease of $35.9 \%$ is found. Finally, the typically adopted locations of the various members of staff during interventions, in accordance with their roles, are found to be an optimal choice. From such findings, we urge the implementation of appropriate in-house protocols in seeking to mitigate the potential deleterious effects of radiation on members of staff.

\section{Conflicts of Interest}

The author declares no conflicts of interest regarding the publication of this paper.

\section{References}

[1] Cousins, C., et al. (2013) ICRP Publication 120: Radiological Protection in Cardiology. Annals of the ICRP, 42, 1-125. https://doi.org/10.1016/j.icrp.2012.09.001

[2] Zakeri, F., Hirobe, T. and Akbari, N.K., (2010) Biological Effects of Low-Dose Ionizing Radiation Exposure on Interventional Cardiologists. Occupational Medicine (London), 60, 464-469. https://doi.org/10.1093/occmed/kqq062

[3] Roguin, A., Goldstein, J., Bar, O. and Goldstein, J. A. (2013) Brain and Neck Tumors among Physicians Performing Interventional Procedures. American Journal of Cardiology, 111, 1368-1372. https://doi.org/10.1016/j.amjcard.2012.12.060

[4] Bogaert, E., Bacher, K. and Thierens, H. (2008) A Large-Scale Multicentre Study in Belgium of Dose Area Product Values and Effective Doses in Interventional Cardiology Using Contemporary X-Ray Equipment. Radiation Protection Dosimetry, 128, 312-323. https://doi.org/10.1093/rpd/ncm379

[5] Faulkner, K., Vano, A., Ortiz, P. and Ruiz, R. (2000) Practical Aspects of Radiation Protection in Interventional Radiology. IRPA-10 Proceedings of the 10 th International Congress of the International Radiation Protection Association on Harmonization of Radiation, Human Life and the Ecosystem, Japan Health Physics Society, 7-16.

[6] Fardid, R., Mirzadeh, F. and Rezaei, H. (2017) Occupational Doses of Cardiologists in Cath Labs and Simulation Method. Journal of Cancer Research and Therapeutics, 13, 901-907.

[7] Baptista, M., et al. (2015) Assessment of the Occupational Exposure in Real Time 
during Interventional Cardiology Procedures. Radiation Protection Dosimetry, 165, 304-309. https://doi.org/10.1093/rpd/ncv052

[8] Toossi, M., et al. (2015) Preliminary Results of an Attempt to Predict over Apron Occupational Exposure of Cardiologists from Cardiac Fluoroscopy Procedures Based on DAP (Dose Area Product) Values. Australasian Physical \& Engineering Sciences in Medicine, 38, 8391. https://doi.org/10.1007/s13246-014-0326-1

[9] Kong, Y., et al. (2015). Influence of Standing Positions and Beam Projections on Effective Dose and Eye Lens Dose of Anaesthetists in Interventional Procedures. Radiation Protection Dosimetry, 163, 181-187. https://doi.org/10.1093/rpd/ncu148

[10] Loy Rodas, N. and Padoy, N., (2015) Seeing Is Believing: Increasing Intraoperative Awareness to Scattered Radiation in Interventional Procedures by Combining Augmented Reality, Monte Carlo Simulations and Wireless Dosimeters. Journal of CARS, 10, 1181-1191. https://doi.org/10.1007/s11548-015-1161-x

[11] Toutaoui, K.N., et al. (2016) Assessment of Radiation Protection of Patients and Staff in Interventional Procedures in Four Algerian Hospitals. Radiation Protection Dosimetry, 168, 55-60. https://doi.org/10.1093/rpd/ncv001

[12] Siiskonen, T., Tapiovaara, M., Kosunen, A., Lehtinen, M. and Vartiainen, E. (2007) Monte Carlo Simulations of Occupational Radiation Doses in Interventional Radiology. The British Journal of Radiology, 80, 460-468. https://doi.org/10.1259/bjr/26692771

[13] Koukoravaa, C., Carinou, E., Ferrari, P., Krim, S. and Struelens, L. (2011) Study of the Parameters Affecting Operator Doses in Interventional Radiology Using Monte Carlo Simulations. Radiation Measurements, 46, 1216-1222. https://doi.org/10.1016/j.radmeas.2011.06.057

[14] Santos, W.S., et al. (2015) Exposures in Interventional Radiology Using Monte Carlo Simulation Coupled with Virtual Anthropomorphic Phantoms. Physica Medica, 31, 929-933. https://doi.org/10.1016/j.ejmp.2015.06.011

[15] Kim, C.H., et al. (2008) HDRK-Man: A Whole-Body Voxel Model Based on High-Resolution Color Slice Images of a Korean Adult Male Cadaver. Physics in Medicine \& Biology, 53, 4093-4106. https://doi.org/10.1088/0031-9155/53/15/006

[16] Allison, J., et al. (2016) Recent Developments in GEANT4. Nuclear Instruments and Methods in Physics Research A, 835, 186-225. https://doi.org/10.1016/j.nima.2016.06.125

[17] White, D.R., Griffth, R.V. and Wilson, I.J. (1992) Photon, Electron, Proton and Neutron Interaction Data for Body Tissues. Journal of the International Commission on Radiation Units and Measurements, os24, Report 46.

[18] Punnoose, J., Xu, J., Sisniega, A., Zbijewski, W. and Siewerdsen, J.H. (2016) Technical Note: Spektr3.0-A Computational Tool for X-Ray Spectrum Modeling and Analysis. Medical Physics, 43, 4711-4717. https://doi.org/10.1118/1.4955438

[19] Efstathopoulos, E.P., et al. (2011) Occupational Radiation Doses to the Extremities and the Eyes in Interventional Radiology and Cardiology Procedures. The British Journal of Radiology, 84, 70-77. https://doi.org/10.1259/bjr/83222759

[20] Cosmo, G. and the Geant4 Collaboration (2014) Geant4-Towards Major Release 10. Journal of Physics: Conference Series, 513, Article ID: 022005. https://doi.org/10.1088/1742-6596/513/2/022005

[21] Klein, O. and Nishina, Y. (1994) On the Scattering of Radiation by Free Electrons According to Dirac's New Relativistic Quantum Dynamics. In: Bethe, H.A. and Guth, A.H., Eds., The Oskar Klein Memorial Lectures, Vol. 2: Lectures, World 
Scientific Publishing, Singapore, 113-139.

https://doi.org/10.1142/9789814335911_0006

[22] ICRU (2005) Patient Dosimetry for X-Rays Used in Medical Imaging, 2005. ICRU Report No. 74, International Commission on Radiation Units and Measurements, Bethesda.

[23] Bethe, A.S., et al. (2006) An Investigation of Operator Exposure in Interventional Radiology. Radiographics, 26, 1533-1541. https://doi.org/10.1148/rg.265055127 